\section{Evolução do aleitamento materno em uma capital da Região Centro-Oeste do Brasil entre 1999 e 2004}

\author{
Breastfeeding trends in a State capital in the \\ Central West region of Brazil, 1999-2004
}

\author{
${ }^{1}$ Coordenadoria de \\ Vigilância Epidemiológica, \\ Secretaria Estadual de \\ Saúde de Mato Grosso, \\ Cuiabá, Brasil. \\ 2 Instituto de Saúde Coletiva, \\ Universidade Federal do \\ Mato Grosso, Cuiabá, Brasil. \\ 3 Instituto de Saúde, \\ Secretaria Estadual de \\ Saúde de São Paulo, \\ São Paulo, Brasil. \\ Correspondência \\ G. S. Brunken \\ Instituto de Saude Coletiva \\ Universidade Federal \\ de Mato Grosso. \\ Av. Fernando Correa da Costa \\ $s / n$, CCBS III, térreo, \\ Cuiabá, MT \\ 78060-900, Brasil. \\ brunken@terra.com.br
}

\begin{abstract}
A cross-sectional study was held on the first day of the National Vaccination Campaign in 2004, in Cuiabá, Mato Grosso State, Brazil, with the objective of identifying breastfeeding evolution from 1999 to 2004 during the first year of life. A two-stage sampling procedure was used, considering the numbers of vaccination units and children in each unit. A nutritional survey with a 24-hour food recall questionnaire was applied to 921 parents or accompanying persons of children less than one year of age. There was an increase in exclusive breastfeeding in all age brackets. At the end of six months, there were low percentages of infants in exclusive breastfeeding (<5\% in 1999 and < 10\% in 2004). The prevalence of exclusive breastfeeding doubled in five years in infants less than four months of age, from $17.7 \%$ to $28.5 \%$. The measures taken to promote breastfeeding were effective, although insufficient in light of the serious situation. More such measures are needed to improve the existing programs.
\end{abstract}

Breast Feeding; Weaning; Nutritional Assessment

\author{
Solanyara Maria da Silva ${ }^{1}$ \\ Gisela Soares Brunken 2 \\ Giovanny Vinícius Araújo de França 2 \\ Maria Mercedes Escuder 3 \\ Sonia Isoyama Venancio ${ }^{3}$
}

\section{Introdução}

O aleitamento materno é um modo insubstituível de fornecer o alimento ideal para o crescimento e desenvolvimento de lactentes, tendo influência biológica, emocional e cognitiva sobre a saúde dos mesmos 1. Com a introdução de modernas tecnologias e adoção de diferentes estilos de vida houve, em muitas sociedades, uma redução notável na importância atribuída à amamentação ${ }^{2}$.

A introdução precoce de outros líquidos, além do leite materno, não é só desnecessária como também prejudicial, mesmo em locais quentes e secos 3,4. O consumo de outros alimentos pode levar à diminuição da freqüência e intensidade da sucção, reduzindo a produção do leite materno 4 . Há também o risco de infecções pela contaminação de mamadeiras ou dos próprios alimentos 5 . Estudos demonstram que ocorre um aumento acentuado na freqüência de diarréia quando o aleitamento materno não é exclusivo 6,7 .

A recomendação mundial preconiza a amamentação exclusiva por seis meses e a manutenção do aleitamento materno complementado até, no mínimo, os dois anos de idade ${ }^{8}$. Muito embora tenham sido observados no Brasil avanços na prática da amamentação, sua manutenção exclusiva ainda está longe do esperado.

Há evidências de melhoria na prática da amamentação exclusiva e da amamentação como um todo, desde a década de 70, evidenciado nos es- 
tudos realizados no Brasil 9,10, especialmente nos estratos sócio-econômicos mais elevados.

Estudo nacional conduzido nas capitais brasileiras em 1999 mostrou que 53,1\% das crianças recebem apenas leite materno no primeiro mês de vida, decaindo o percentual conforme aumenta a idade. Comparando-se as capitais e o Distrito Federal, a melhor situação na modalidade de aleitamento exclusivo para os menores de quatro meses, encontra-se em Fortaleza, Estado do Ceará $(57,1 \%)$, enquanto Cuiabá, Estado do Mato Grosso, apresenta a menor taxa $(17,7 \%)$. A mediana do aleitamento materno exclusivo e de aleitamento materno no Brasil eram, respectivamente, de 23,4 dias e 295,9 dias 11 . No caso de Cuiabá, verificou-se um desempenho bom em relação ao aleitamento materno (mediana de 357,1 dias) quando comparado a outras capitais, mas a mediana de aleitamento materno exclusivo foi a pior entre todas (5,1 dias), sendo classificada a primeira capital em desmame precoce 11. Diante dessa situação alarmante, algumas ações foram desenvolvidas para reverter o quadro, e faz-se necessária uma nova investigação para conhecer a evolução da amamentação entre 1999 e 2004, avaliando as ações existentes, e possibilitando traçar novas estratégias para incentivo ao aleitamento materno exclusivo.

\section{Material e métodos}

Realizou-se inquérito sobre práticas alimentares por ocasião do primeiro dia da Campanha Nacional de Vacinação de 2004 (5 de junho) em Cuiabá, por meio de uma parceria entre o Instituto de Saúde da Secretaria Estadual de Saúde de São Paulo (Projeto Amamentação e Municípios) e a Universidade Federal de Mato Grosso.

A projeção de menores de um ano para o ano de 2004 foi de 8.129 crianças, com base no método de progressão aritmética 12 , calculado com base nos censos demográficos de 1991 e 2000. Planejou-se uma amostra de 1.040 crianças, suficiente para estimar a prevalência de aleitamento materno para as diferentes faixas etárias com um nível de significância de 95\%. Adotou-se o procedimento de amostragem por conglomerado, com sorteio em dois estágios, com probabilidade proporcional ao tamanho dos conglomerados, devido ao fato de que as crianças não se distribuem uniformemente nas unidades de vacinação. Assim, todas as crianças que foram aos postos de vacinação na campanha tiveram igual probabilidade de pertencer à amostra sorteada. No primeiro estágio foram sorteadas 29 Unidades de Vacinação das 60 selecionadas para campanha, mas uma delas foi sorteada duas vezes, por conta do número de crianças cadastradas. No segundo estágio foram sorteadas as crianças em cada unidade, de forma sistemática, obedecendo à fração de sorteio anteriormente calculada.

O instrumento utilizado foi elaborado pelo Instituto de Saúde, mediante recomendações da Organização Mundial da Saúde para inquéritos sobre amamentação ${ }^{13}$. Trata-se de questionário semi-estruturado, organizado em blocos referentes ao aleitamento materno e alimentação infantil nas últimas 24 horas, características das crianças e das mães. Para caracterização do padrão de aleitamento materno adotou-se o seguinte 13:

- Aleitamento materno exclusivo: a criança recebe apenas leite materno, de sua mãe ou amade-leite, ou leite ordenhado, e não recebe outros líquidos ou sólidos com exceção de suplementos minerais, vitaminas ou medicamentos;

- Aleitamento materno predominante: a fonte predominante de nutrição da criança é o leite materno, porém também pode receber água e bebidas a base de água (água açucarada ou com sabores, infusões, chás etc.), suco de frutas, soluções de reidratação oral (SRO), vitaminas, minerais e medicamentos em gotas e xaropes, e líquidos cerimoniais (em quantidades limitadas); - Aleitamento materno: a criança recebe leite materno (diretamente do peito ou ordenhado) independente da ingestão de alimentos sólidos, semi-sólidos e líquidos, incluindo leite artificial.

Realizou-se reunião de sensibilização com os gerentes das unidades selecionados para o estudo, abordando-se a importância da pesquisa para verificar a evolução do perfil de amamentação no município. Os questionários foram aplicados por estudantes de Nutrição, Enfermagem e Medicina, que receberam treinamento sobre o objetivo e metodologia da pesquisa, amostragem, organização da coleta de dados, aplicação do instrumento de coleta, bem como Manual do Entrevistador, a fim de padronizar condutas de campo.

As mães ou acompanhantes das crianças potencialmente participantes da amostra foram abordadas na fila de vacinação e, uma vez informadas sobre o objetivo da pesquisa e aceitando participar, iniciava-se a entrevista.

Foram calculadas as freqüências, intervalos de confiança e variação na prevalência do aleitamento materno, aleitamento materno exclusivo e aleitamento materno predominante por faixa etária intervalar de trinta dias entre os anos de 1999 e 2004, utilizando-se o programa Csample do Epi Info 6.04 (Centers for Disease Control and Prevention, Atlanta, Estados Unidos). Com esse mesmo programa, foi possível analisar o efeito do desenho amostral, o deff sobre as estimativas obtidas. 
Para avaliar a evolução do aleitamento materno em suas três modalidades, foi utilizado o delta da prevalência do aleitamento $(\Delta \%)$, calculado pela diferença dos anos, dividindo-se pelo valor de 1999. Foi considerada variação significativa quando não houve sobreposição dos intervalos de confiança.

O projeto foi aprovado pelo Comitê de Ética em Pesquisa do Hospital Universitário Julio Müller. Os gerentes das Unidades Básicas de Saúde envolvidos no estudo assinaram termo de consentimento livre e esclarecido. Os acompanhantes das crianças potencialmente da amostra eram informados sobre os objetivos da pesquisa e solicitado consentimento verbal para aplicação do inquérito.

\section{Resultados}

No dia "D" da campanha de vacinação (5 de junho de 2004), compareceram 7.300 crianças, configurando-se uma alta cobertura $(89,8 \%)$. Desse total, 921 compõem a amostra do estudo.

A Tabela 1 apresenta as características da amostra, sejam elas relacionadas à mãe ou à criança. As crianças da amostra distribuíram-se uniformemente em relação ao sexo e idade em meses. Uma pequena parte nasceu com baixo peso $(6 \%)$ e a maioria $(57,4 \%)$ nasceu de parto cesáreo. Poucas crianças $(6,3 \%)$ vacinadas em Cuiabá haviam nascido em outros municípios. Em relação às características maternas, quase todas as acompanhantes das crianças $(83,9 \%)$ eram as próprias mães. Uma parcela $(16,4 \%)$ era adolescente $(<20$ anos) e a quinta parte não havia completado a 4a série do 1o grau $(24,6 \%)$ e 1,7\% relatou não ter instrução. No outro extremo do nível de instrução, quase a metade $(46,4 \%)$ havia completado o 2 o grau ou tinha o 3o grau, completo ou não. Cerca de metade das mães informou que a criança era o seu primeiro filho.

As Tabelas 2, 3 e 4 apresentam a evolução das modalidades de aleitamento materno por faixa etária, no período entre 1999 e 2004 . Houve incremento consistente na freqüência de aleitamento materno nas faixas etárias de menores de um mês $(8,7 \%)$ e de maiores de nove meses $(21,1 \%)$. Ainda que tenha havido um pequeno incremento apenas nessas faixas etárias, percebe-se que, ao final do primeiro ano de vida, $40 \%$ das crianças já não recebiam leite materno.

Entre as crianças menores de 180 dias, parece haver uma tendência, em todas as idades, de ligeiro decréscimo da prática de aleitamento materno predominante em relação à pesquisa de 1999.
Tabela 1

Características das crianças e das mães em pesquisa de aleitamento materno.

Cuiabá, Mato Grosso, Brasil, 2004

\begin{tabular}{|c|c|c|}
\hline Características & $\mathrm{n}$ & $\%$ \\
\hline \multicolumn{3}{|l|}{ Infantis } \\
\hline \multicolumn{3}{|l|}{ Sexo } \\
\hline Masculino & 478 & 51,9 \\
\hline Feminino & 443 & 48,1 \\
\hline \multicolumn{3}{|l|}{ Idade (dias) } \\
\hline 0 아인 & 61 & 6,6 \\
\hline $30+60$ & 71 & 7,7 \\
\hline 60 -90 & 77 & 8,4 \\
\hline 90 -120 & 86 & 9,3 \\
\hline 120 -150 & 70 & 7,6 \\
\hline 150 -180 & 76 & 8,3 \\
\hline 180 - 270 & 215 & 23,3 \\
\hline $270+365$ & 265 & 28,8 \\
\hline \multicolumn{3}{|l|}{ Peso ao nascer (g) } \\
\hline$<2.500$ & 55 & 6,0 \\
\hline 2.500 - 3.000 & 205 & 22,3 \\
\hline$\geq 3.000$ & 644 & 69,9 \\
\hline Em branco & 17 & 1,8 \\
\hline \multicolumn{3}{|l|}{ Local de nascimento } \\
\hline Cuiabá & 863 & 93,7 \\
\hline Outros municípios & 58 & 6,3 \\
\hline \multicolumn{3}{|l|}{ Tipo de parto } \\
\hline Vaginal & 386 & 41,6 \\
\hline Cesárea & 529 & 57,4 \\
\hline Não sabe/Em branco & 9 & 1,0 \\
\hline \multicolumn{3}{|l|}{ Maternas } \\
\hline \multicolumn{3}{|l|}{ Acompanhante da criança } \\
\hline Sim & 773 & 83,9 \\
\hline \multicolumn{3}{|l|}{ Idade (anos) } \\
\hline$<20$ & 127 & 16,4 \\
\hline $20+35$ & 588 & 76,1 \\
\hline$\geq 30$ & 58 & 7,5 \\
\hline \multicolumn{3}{|l|}{ Escolaridade } \\
\hline Sem instrução & 13 & 1,7 \\
\hline 1ㅇ grau incompleto & 190 & 24,6 \\
\hline 1o grau completo & 68 & 8,8 \\
\hline 2o grau incompleto & 111 & 14,4 \\
\hline 2o grau completo & 255 & 33,0 \\
\hline 3o grau & 103 & 13,4 \\
\hline Em branco & 33 & 4,3 \\
\hline \multicolumn{3}{|l|}{ Trabalha fora de casa } \\
\hline Sim & 193 & 25,1 \\
\hline \multicolumn{3}{|l|}{ Primípara } \\
\hline Sim & 341 & 44,1 \\
\hline
\end{tabular}


Tabela 2

Prevalências e intervalos de confiança (IC95\%) do aleitamento materno, segundo faixa etária das crianças menores de um ano. Cuiabá, Mato Grosso, Brasil.

\begin{tabular}{|c|c|c|c|c|c|}
\hline \multirow[t]{2}{*}{ Idade (dias) } & \multicolumn{2}{|c|}{$1999(a)$ * } & \multicolumn{2}{|c|}{2004 (b) } & \multirow[t]{2}{*}{$\Delta(\%) \star \star$} \\
\hline & $\%$ & IC95\% & $\%$ & IC95\% & \\
\hline $0-30$ & 90,5 & $88,4-92,3$ & 98,4 & $95,1-101,7$ & $+8,7$ \\
\hline $31-60$ & 88,6 & $86,4-90,5$ & 91,0 & $85,3-96,5$ & $+2,7$ \\
\hline $61-90$ & 86,4 & $84,1-88,3$ & 89,5 & $83,4-95,6$ & $+3,6$ \\
\hline $91-120$ & 83,9 & $81,6-85,8$ & 90,5 & $84,3-96,7$ & $+7,9$ \\
\hline $121-150$ & 80,8 & $78,6-82,8$ & 77,5 & $65,7-89,2$ & $-4,1$ \\
\hline $151-180$ & 77,5 & $75,3-79,4$ & 75,3 & $65,4-85,3$ & $-2,8$ \\
\hline $181-270$ & 65,0 & $62,7-67,3$ & 64,7 & $59,0-70,3$ & $-0,5$ \\
\hline $271-365$ & 49,4 & $45,7-53,2$ & 59,8 & $54,1-65,5$ & $+21,1$ \\
\hline Total de crianças & \multicolumn{2}{|c|}{2.186} & \multicolumn{2}{|c|}{921} & - \\
\hline
\end{tabular}

* Fonte: Pesquisa de Prevalência do Aleitamento Materno nas Capitais e no Distrito Federal 11;

** $\frac{(\mathrm{b}-\mathrm{a})}{\mathrm{a}} \times 100$.

Tabela 3

Prevalências e intervalos de confiança (IC95\%) do aleitamento materno predominante, segundo idades das crianças menores de 180 dias.

Cuiabá, Mato Grosso, Brasil.

\begin{tabular}{|c|c|c|c|c|c|}
\hline \multirow[t]{2}{*}{ Idade (dias) } & \multicolumn{2}{|c|}{1999 (a) * } & \multicolumn{2}{|c|}{2004 (b) } & \multirow[t]{2}{*}{$\Delta(\%) * \star$} \\
\hline & $\%$ & IC95\% & $\%$ & IC95\% & \\
\hline $0-30$ & 45,2 & $39,3-51,2$ & 44,3 & $28,4-60,1$ & -2 \\
\hline $31-60$ & 39,1 & $34,9-43,5$ & 32,5 & $22,4-42,5$ & -17 \\
\hline $61-90$ & 33,3 & $30,3-36,5$ & 29,0 & $19,4-38,5$ & -12 \\
\hline $91-120$ & 28,0 & $25,3-31,0$ & 29,8 & $18,7-40,8$ & +6 \\
\hline $121-150$ & 23,3 & $20,2-26,7$ & 21,1 & $14,1-28,1$ & -9 \\
\hline $151-180$ & 19,1 & $15,6-23,2$ & 12,5 & $4,7-20,3$ & -34 \\
\hline
\end{tabular}

* Fonte: Pesquisa de Prevalência do Aleitamento Materno nas Capitais e no Distrito Federal 11.

** $\underline{(b-a)} \times 100$.

O grande incremento ocorreu, efetivamente, em relação à modalidade de aleitamento materno exclusivo, ocorrendo incremento positivo em todas as faixas etárias, mesmo que somente nas idades entre maiores de 60 e menores de 120 dias essa diferença seja estatisticamente significante. Em ambos os estudos, chega-se ao final dos 180 dias com menos de $5 \%$ (em 1999) e de $10 \%$ (em 2004) das crianças mamando exclusivamente no peito.

A Tabela 5 compara as duas modalidades de aleitamento (aleitamento materno e aleitamento materno exclusivo) em menores de 120 dias, entre os dois períodos investigados. Percebe-se uma nítida melhoria na evolução dessa prática.

\section{Discussão}

No tocante à validade interna, adotaram-se diversos procedimentos a fim de garantir a confiabilidade nos resultados apresentados. Houve treinamento de quatro horas dos entrevistadores e supervisores de campo, que receberam Manual de Entrevistador e Manual de Supervisor, promovendo uniformidade de abordagem no inquérito aplicado às mães na fila de vacinação. Cada unidade de vacinação contou com um supervisor e os dois coordenadores do projeto estiveram em 27 dos 29 postos de vacinação no dia “D”, e contavam com telefones móveis que permitiam resolver prontamente qualquer dúvida no trabalho 
Prevalências e intervalos de confiança (IC95\%) do aleitamento materno exclusivo, segundo idades das crianças menores de 180 dias.

Cuiabá, Mato Grosso, Brasil.

\begin{tabular}{|c|c|c|c|c|c|}
\hline \multirow[t]{2}{*}{ Idade (dias) } & \multicolumn{2}{|c|}{1999 (a) * } & \multicolumn{2}{|c|}{2004 (b) } & \multirow[t]{2}{*}{$\Delta(\%)$ ** } \\
\hline & $\%$ & IC95\% & $\%$ & IC95\% & \\
\hline $0-30$ & 29,7 & $3,9-36,1$ & 45,9 & $31,6-60,2$ & $+54,5$ \\
\hline $31-60$ & 20,7 & $17,4-24,5$ & 24,7 & $15,4-34,0$ & $+19,3$ \\
\hline $61-90$ & 14,0 & $11,8-16,4$ & 26,3 & $17,0-35,7$ & $+87,9$ \\
\hline $91-120$ & 9,1 & $7,3-11,3$ & 21,4 & $13,2-29,6$ & $+135,2$ \\
\hline $121-150$ & 5,9 & $4,3-8,0$ & 7,0 & $1,6-12,5$ & $+18,6$ \\
\hline $151-180$ & 3,7 & $2,4-5,6$ & 6,9 & $1,8-12,1$ & $+86,5$ \\
\hline
\end{tabular}

* Fonte: Pesquisa de Prevalência do Aleitamento Materno nas Capitais e no Distrito Federal 11;

** $\frac{(b-a)}{a} \times 100$.

Tabela 5

Prevalências do aleitamento materno, aleitamento materno exclusivo e intervalos de confiança (IC95\%), em crianças menores de 120 dias.

Cuiabá, Mato Grosso, Brasil.

\begin{tabular}{|c|c|c|c|c|c|}
\hline \multirow[t]{2}{*}{ Modalidades do aleitamento } & \multicolumn{2}{|c|}{$1999(a)$ * } & \multicolumn{2}{|c|}{2004 (b) } & \multirow[t]{2}{*}{$\Delta(\%)$ ** } \\
\hline & $\%$ & IC95\% & $\%$ & IC95\% & \\
\hline Aleitamento materno & 89,1 & $86,4-91,2$ & 92,2 & $89,3-95,1$ & $+3,5$ \\
\hline Aleitamento materno exclusivo & 17,7 & $15,0-20,9$ & 28,5 & $22,9-34,0$ & $+61,0$ \\
\hline
\end{tabular}

* Fonte: Pesquisa de Prevalência do Aleitamento Materno nas Capitais e no Distrito Federal 11;

** $\frac{(b-a)}{a} \times 100$.

de campo. Essas estratégias contribuíram para garantir a padronização e qualidade dos dados coletados.

A cobertura vacinal em Cuiabá, no primeiro dia da primeira fase da Campanha de Vacinação em 2004 foi relativamente alta $(89,8 \%)$, sendo próxima à encontrada no estudo das capitais e Distrito Federal de 1999. Conforme relatório do Programa Nacional de Imunização, naquele ano de 1999 a cobertura vacinal variou de $88,5 \%$ a $100 \% 11$. O processo de seleção da amostra, utilizando a técnica de conglomerados em duas etapas, permite que cada criança presente no dia de vacinação tenha igual chance de participar da pesquisa. A avaliação do efeito do desenho permitiu observar que a precisão dos resultados apresentou-se semelhante àquela alcançada com uma amostra do mesmo tamanho obtida pelo processo causal simples.
A principal evolução, nesses cinco anos, foi um incremento maior que $50 \%$ do aleitamento materno exclusivo em menores de quatro meses, especialmente nos terceiro e quarto meses de idade. No entanto, observa-se baixa freqüência em crianças menores de um mês $(<50 \%)$ e maiores de quatro meses $(<10 \%)$, permanecendo muito aquém das recomendações estabelecidas 8,13 . Outro importante achado foi a manutenção do aleitamento materno, embora não na modalidade exclusiva, em torno de $90 \%$ em menores de 120 dias.

É possível que não tenha havido variação significativa no aleitamento materno predominante porque houve, na verdade, melhoria no aleitamento materno exclusivo e estabilidade no aleitamento materno de forma geral. $\mathrm{O}$ aleitamento materno predominante não é a meta de nenhum programa de incentivo ao aleitamento, mas refle- 
te uma fase transitória de introdução de líquidos não nutritivos que culturalmente está presente no Brasil, particularmente em Cuiabá, que apresenta uma elevada temperatura e baixa umidade relativa do ar.

As ações realizadas em Cuiabá, pelas três esferas do governo, nesses últimos cinco anos, surtiram resultados positivos na prática da amamentação. Dentre tais ações, pode-se citar a inauguração de três bancos de leite nos principais hospitais públicos da cidade, quando antes não havia nenhum. Houve, também, capacitações para todos os agentes comunitários de saúde e de profissionais tais como técnicos de enfermagem dos hospitais e pronto-socorro municipal, além de maior divulgação do aleitamento materno nos diversos meios de comunicação e realização de eventos a respeito do tema. Sobre esse último aspecto, deve-se ressaltar como principal momento o VIII Encontro Nacional de Aleitamento Materno em Cuiabá em 2003, com participação maciça dos profissionais de saúde, gestores, estudantes, pesquisadores.

Programas de capacitação da equipe de saúde têm demonstrado grande impacto nas práticas desses profissionais, levando a uma maior duração da amamentação nas comunidades atendidas 14,15. A importância da prática e conhecimento do aconselhamento em amamentação pelos profissionais de saúde constitui um poderoso instrumento de intervenção para o aumento das taxas e duração do aleitamento materno 16 .

Infelizmente, percebe-se que há muito que se avançar nessas intervenções. A capital do Estado de Mato Grosso ainda não possui nenhum Hospital Amigo da Criança, embora tenham iniciativas concretas nesse sentido nos três hospitais públicos que possuem bancos de leite. O único Hospital Amigo da Criança no Estado, que era particular e distante da capital, foi fechado em 2005 por problemas financeiros. O nascimento em Hospital Amigo da Criança é reconhecidamente um fator de proteção ao aleitamento ma- terno 17, mas o fato de existir Hospital Amigo da Criança no município também está relacionado à proteção do aleitamento materno ${ }^{9}$, pois as crianças que nascem em município sem Hospital Amigo da Criança têm 2,2 vezes mais chance de receber outro alimento precocemente.

Ações como o monitoramento periódico da Norma Brasileira de Comercialização de Alimentos para Lactentes 18 pela Superintendência de Defesa do Consumidor de Mato Grosso (PROCON-MT) e pela Vigilância Sanitária, além da maior divulgação na mídia sobre os efeitos nocivos da introdução precoce de líquidos como água, suco e chá para crianças menores de seis meses em aleitamento materno exclusivo, ajudam no controle do uso indiscriminado dos substitutos do leite materno.

Outra ação comprovadamente eficaz seria a adoção da Iniciativa Unidade Básica Amiga da Amamentação. Tem como princípio que toda Unidade Básica de Saúde (como os postos de saúde, os centros de saúde, os postos de saúde da família etc.) que tenha pré-natal e pediatria pode tornar-se uma Unidade Básica Amiga da Amamentação. Para isso, deve cumprir os Dez Passos para o Sucesso da Amamentação. Esses passos definem intervenções a serem conduzidas nas fases de pré-natal e acompanhamento do binômio mãe-bebê e foram efetivas em estender a duração da amamentação 19 .

As ações realizadas foram efetivas, contudo, insuficientes para a grave situação em relação ao aleitamento materno exclusivo. Sugere-se, então, que sejam mantidas as ações em curso, e que se implementem novos projetos, como implantação de hospitais e de unidades básicas de saúde "amigas da criança”, treinamento prático de todos os profissionais de saúde que lidam com o pré e pós-natal, implantação de grupos de apoio ao aleitamento materno e vigilância da Lei $n^{o}$. 11.26518 , que regulamenta a comercialização de alimentos para lactentes e crianças de primeira infância. 


\section{Resumo}

Um estudo de caráter transversal foi realizado durante o primeiro dia da Campanha Nacional de Vacinação de 2004, em Cuiabá, Mato Grosso, Brasil, com o objetivo de conhecer a evolução do aleitamento materno entre 1999 e 2004, no primeiro ano de vida. A determinação da amostra seguiu dois passos, considerando o número de unidades de vacinação e as crianças em cada unidade. Um inquérito nutricional contendo um recordatório alimentar de 24 horas foi aplicado a 921 acompanhantes das crianças menores de um ano. Houve incremento positivo em todas as faixas etárias na modalidade de aleitamento materno exclusivo. Observou-se, ao final dos 180 dias, baixo percentual de crianças em aleitamento materno exclusivo, sendo menos de 5\% (em 1999) e de 10\% (em 2004). A prevalência do aleitamento materno exclusivo dobrou em cinco anos nas crianças menores de 120 dias, passou de $17,7 \%$ para $28,5 \%$. As ações realizadas para promoção do aleitamento materno foram efetivas, porém, insuficientes para a grave situação. Mais ações são necessárias, bem como o aperfeiçoamento dos programas existentes.

Aleitamento Materno; Desmame; Avaliação Nutricional;

\section{Referências}

1. Akrè J. Alimentação infantil: bases fisiológicas. Genebra: Organização Mundial da Saúde/Rede Internacional em Defesa do Direito de Amamentar/Instituto de Saúde; 1994.

2. Rea MF. Substitutos do leite materno: passado e presente. Rev Saúde Pública 1990; 24:241-9.

3. Ashraf RN, Jalil F, Aperia A, Lindblad BS. Additional water is not needed for healthy breastfed babies in a hot climate. Acta Pediatr 1993; 82:7-11.

4. Sachdev HPS, Krishna J, Puri RK, Satyanarayana L, Kumar S. Water supplementation in exclusively breastfed infants during summer in the tropics. Lancet 1991; 337:929-33.

5. Horta BL, Olinto MTA, Victora CG, Barros FC, Guimarães PRV. Amamentação e padrões alimentares em crianças de duas coortes de base populacional no sul de Brasil: tendências e diferenciais. Cad Saúde Pública 1996; 12 Suppl 1:43-8.

\section{Colaboradores}

S. M. Silva, G. S. Brunken e G. V. A. França participaram na realização do trabalho em campo, no processamento e análise dos dados e na redação do artigo. M. M. Escuder e S. I. Venancio colaboraram na organização da pesquisa e na revisão crítica do artigo.

\section{Agradecimentos}

Ao Instituto de Saúde da Secretaria Estadual de Saúde de São Paulo, pela assessoria no delineamento do estudo e acompanhamento. Às Secretaria Estadual de Saúde de Mato Grosso e Secretaria Municipal de Saúde de Cuiabá pelo consentimento para realização da pesquisa e financiamento. Aos acadêmicos das faculdades de Nutrição, Medicina e Enfermagem da Universidade Federal de Mato Grosso, pelo auxílio na coleta de dados, bem como aos profissionais que participaram como supervisores de campo. Ao Conselho Nacional de Desenvolvimento Científico e Tecnológico (CNPq) pelas bolsas de mestrado e iniciação científica concedidas.
6. Giugliani ERJ. Aleitamento materno na prática clínica. J Pediatr (Rio de J) 2000; 76 Suppl:238-52.

7. Vieira GO, Silva LR, Vieira TO. Alimentação infantil e morbidade por diarréia. J Pediatr (Rio de J) 2003; 79:449-54.

8. Monteiro CA, Zuñiga HPP, Benicio MHd'A, Rea MF. Estudo das condições de saúde das crianças do Município de São Paulo, SP (Brasil), 1984-1985. III. Aleitamento materno. Rev Saúde Pública 1987; 21: 13-22.

9. Venancio SI, Monteiro CA. A evolução da prática da amamentação nas décadas de 70 e 80 . Rev Bras Epidemiol 1998; 1:40-9.

10. World Health Organization. Report of the expert consultation on the optimal duration of exclusive breastfeeding. Geneva: World Health Organization; 2001. 
11. Ministério da Saúde. Pesquisa de prevalência do aleitamento materno nas capitais e no Distrito Federal. Brasília: Ministério da Saúde; 2001.

12. Laurenti R, Gotlieb SL, Mello-Jorge MH, Lebrão ML. Estatísticas de Saúde. São Paulo: E.P.U.; 1987.

13. Organización Mundial de la Salud. Indicadores para evaluar las prácticas de lactancia materna. Geneva: Organización Mundial de la Salud; 1991.

14. Neylor A, Wester R. Providing professional lactation management consultation. Clin Perinatol 1987; 14:33-50.

15. Valdés V, Pérez A, Labbok M, Pugin E, Zambranco I, Catalan S. The impact of a hospital and clinicbased breastfeeding promotion program in a middle class urban environment. J Trop Pediatr 1993, 39:142-51.
16. Bueno LGS, Teruya KM. Aconselhamento em amamentação e sua prática. J Pediatr (Rio de J) 2004; 80 Supl 5:126-30.

17. Lutter C, Perez-Escamilla R, Segall A, Sanghvi T, Teruya K, Wichham C. The effectiveness of a hospital-based program to promote exclusive breastfeeding among low-income women in Brazil. Am J Public Health 1997; 87:659-63.

18. Brasil. Lei $n^{\circ}$. 11.265. Regulamenta a comercialização de alimentos para lactentes e crianças de primeira infância. Diário Oficial da União 2006; 4 jan.

19. De Oliveira MIC, Camacho LAB. Impacto das unidades básicas de saúde na duração do aleitamento materno exclusivo. Rev Bras Epidemiol 2002; 5:41-51.

Recebido em 20/Dez/2005

Versão final reapresentada em 27/Dez/2006

Aprovado em 10/Jan/2007 